44-2 | 2019

Varia

\title{
Lisa FitzGerald, Re-Place. Irish Theatre Environments
}

\section{Martine Pelletier}

\section{OpenEdition}

1 Journals

\section{Édition électronique}

URL : https://journals.openedition.org/etudesirlandaises/8677

DOI : 10.4000/etudesirlandaises.8677

ISSN : 2259-8863

\section{Éditeur}

Presses universitaires de Caen

\section{Édition imprimée}

Date de publication : 31 décembre 2019

Pagination : 158-159

ISBN : 978-2-84133-964-8

ISSN : 0183-973X

\section{Référence électronique}

Martine Pelletier, «Lisa FitzGerald, Re-Place. Irish Theatre Environments », Etudes irlandaises [En ligne], 44-2 | 2019, mis en ligne le 06 mai 2020, consulté le 15 novembre 2022. URL : http://

journals.openedition.org/etudesirlandaises/8677; DOI : https://doi.org/10.4000/etudesirlandaises 8677

\section{(c) (1) (2)(2)}

Creative Commons - Attribution - Pas d'Utilisation Commerciale - Partage dans les Mêmes Conditions 4.0 International - CC BY-NC-SA 4.0

https://creativecommons.org/licenses/by-nc-sa/4.0/ 
Pier Luigi Tazzi (p. 124-126) also evokes the threads that connect the artist to "the Tuscany of myth" and Ireland while underlining the artist's independent solitude and feminine sensibility.

This collection of texts does not document the artist's works and processes, nor does it dissolve the fascinating mystery surrounding her pieces but it gives multiple insights into the singularity of her imaginary world. It is therefore an invitation to discover, or rediscover, Mullarney's sculptures and installations, to delve into the geographical hybridity and the profound emotional beauty emanating from her uncanny figures, to embrace the cultural and human continuity her works celebrate.

Valérie MoRIsson

\section{Lisa FitzGerald, Re-Place. Irish Theatre Environments, Oxford, P. Lang, 2017, 202 p.}

Publié dans la série «Reimagining Ireland» de Peter Lang, cet ouvrage de Lisa FitzGerald s'inscrit dans un courant écocritique en plein essor, témoignant de ce tournant «spatial» bien sensible dans les études littéraires et culturelles depuis le début des années 2000. Après la domination des mots, avec le célèbre tournant linguistique des années 1980, c'est désormais l'espace dans toutes ses acceptions qui fascine critiques et praticiens. Le livre reprend le travail de doctorat de Lisa FitzGerald, jeune chercheuse qui après sa thèse préparée à Galway enseigne désormais en Allemagne au Rachel Carson Centre for Environment and Society.

Lisa FitzGerald s'attache à définir et analyser ce qu'elle nomme des «environnements théâtraux conceptuels», un concept lui-même parfois un peu difficile à cerner. Le livre permet de découvrir le travail d'une chercheuse engagée et passionnée, qui pose sa voix au carrefour de diverses disciplines (humanités environnementales et numériques, études théâtrales...) avec une approche critique très "matérialiste» d'un objet en lui-même assez peu original puisque les œuvres choisies dans le répertoire du théâtre irlandais sont les pièces de Synge, All That Fall et Not I de Beckett, Translations et Making History de Brian Friel. Le dernier chapitre ouvre une réflexion très opportune sur l'évolution du concept même de théâtre ou de performance avec l'avènement de multiples médiations et captations numériques qui remettent fondamentalement en cause la coprésence acteur / spectateurs comme définition première et indépassable du théâtre façon Peter Brook.

Le premier chapitre, intitulé «Synge and the Emergence of Irish Ecodrama», revisite les pièces de Synge à la lumière de ses textes ethnographiques et du projet DruidSynge. Les paysages naturels de l'Ouest irlandais, nous rappelle l'auteure, agissent comme représentations de l'identité nationale et la décision de la compagnie Druid et de son metteur en scène Gary Hynes de jouer Synge sur les îles d'Aran était une forme de performance de la mémoire théâtrale et culturelle de l'Ouest et de toute l'Irlande, une exploration de l'idée même d'authenticité si cruciale dans 
l'histoire de l'Irish Cultural Revival. Le chapitre suivant se tourne vers la ville et le corps avec le théâtre de Beckett et propose une belle analyse, fortement appuyée sur les écrits critiques de Stacy Alaimo et son concept de trans-corporalité, du corps de Maddy Rooney, protagoniste de All That Fall, comme non seulement stérile mais aussi en plein échange de matière avec son environnement dans un processus de dissolution / liquéfaction. Lisa FitzGerald analyse les versions données par Pan Pan de plusieurs pièces de Beckett et la manière dont le son de ces pièces écrites pour la radio devient performance dans ces productions sur scène.

Après l'Ouest et Dublin, c'est vers l'espace contesté du Nord que Lisa FitzGerald tourne son attention à travers Field Day et deux pièces de Brian Friel, Translations et Making History. L'auteure s'appuie pour son analyse d'une production théâtrale non sur les productions originales mais sur la production in situ de Making History par Ourouboros qui a beaucoup tourné en Irlande avec l'aide de l'Office of Public Works mais a également été donnée au Centre culturel irlandais de Paris lors de la manifestation «Friel in Paris» en 2009. À l'évidence, jouer cette pièce dont le héros est Hugh O’Neill à Dungannon, dans le lieu où le dernier grand chef gaélique avait son château, permet, comme l'affirme Lisa FitzGerald, de confronter directement le spectateur à l'histoire dans la matérialité d'un lieu que le théâtre ranime.

Le dernier chapitre est certainement le plus original et ouvre des perspectives passionnantes autour de la question des archives numériques, de plus en plus nombreuses et variées, et de leur statut car ces objets à la matérialité ambivalente (réseau, espaces numériques) remettent en cause le caractère éphémère et non reproductible de l'acte théâtral; dans le cadre théorique matérialiste qui est le sien, l'auteure plaide pour une inclusion dans l'analyse de ce qui demeure après l'événement, de ce que la représentation laisse comme documentation d'elle-même et de sa conception.

L'immense mérite de ce travail est d'illustrer de manière concrète dans le domaine de la critique théâtrale ce qui se joue depuis de nombreuses années dans la pratique, avec le développement de formes théâtrales de plus en plus hybrides, sans oublier tout le mouvement in situ qui dans ses formes les plus abouties fait dialoguer espace et acteurs, humain et non-humain, objet culturel et environnement naturel. La réflexion proposée nous engage à re-placer ou rem-placer certains modes de lecture et d'analyse, à regarder différemment la relation nature / culture à la lumière de la crise environnementale qui interroge la place du vivant dans nos sociétés.

Martine Pelletier

\section{Reporting the Troubles: Journalists Tell Their Stories of the Northern Ireland Conflict, Deric Henderson, Ivan Little (eds.), Newtownards, Blackstaff Press, 2018, 238 p.}

Reporting the Troubles is the latest addition to a number of collections of testimonial narratives, both a genre and a practice that developed in the aftermath of the Good 